\title{
PARTIAL DIFFERENTIAL EQUATIONS FOR PRODUCTS OF TWO CLASSICAL ORTHOGONAL POLYNOMIALS
}

\author{
D. W. LEE
}

\begin{abstract}
We give a method to derive partial differential equations for the product of any two classical orthogonal polynomials in one variable and thus find several new differential equations. We also explain with an example that our method can be extended to a more general case such as product of two sets of orthogonal functions.
\end{abstract}

\section{Introduction}

The classical orthogonal polynomials in one variable such as Jacobi polynomials, Bessel polynomials, Laguerre polynomials, and Hermite polynomials, have a various common properties such as Rodrigues' formula, Pearson type equations for orthogonalizing weights, Hahn's property, raising recurrence relations and so on. We refer to $[2,5,9]$ and references therein. One of the most important properties for classical orthogonal polynomials in one variable is that they satisfy a second order differential equation of the form

$$
a_{2}(x) y^{\prime \prime}+a_{1}(x) y^{\prime}=\lambda_{n} y
$$

where $a_{2}(x)$ and $a_{1}(x)$ are polynomials of degree $\leq 2$ and $\leq 1$, respectively, and $\lambda_{n}$ is the eigenvalue parameter. See $[1,5,7]$ for more details. More precisely, by a linear change of variable, we have that

Received October 15, 2003. Revised December 20, 2003.

2000 Mathematics Subject Classification: 33C50, 35P99.

Key words and phrases: orthogonal polynomials, differential equation, classical orthogonal polynomials. (2003).

This work was supported by Kyungpook National University Research Fund 
(i) The Jacobi polynomials $\left\{P_{n}^{(\alpha, \beta)}(x)\right\}_{n=0}^{\infty}(\alpha, \beta, \alpha+\beta+1 \notin\{-1,-2$, $\cdots\})$ satisfy

$\left(1-x^{2}\right) y^{\prime \prime}(x)+[\beta-\alpha-(\alpha+\beta+2) x] y^{\prime}(x)=-n(n+\alpha+\beta+1) y(x)$.

(ii) The Bessel polynomials $\left\{B_{n}^{(d, e)}(x)\right\}_{n=0}^{\infty}(e \neq 0$ and $d \notin\{0,-1,-2$, $\cdots\}$ ) satisfy

$$
x^{2} y^{\prime \prime}(x)+(d x+e) y^{\prime}(x)=n(n+d-1) y(x) .
$$

(iii) The Laguerre polynomials $\left\{L_{n}^{(\alpha)}(x)\right\}_{n=0}^{\infty}(\alpha \notin\{-1,-2, \cdots\})$ satisfy

$$
x y^{\prime \prime}(x)+(\alpha+1-x) y^{\prime}(x)=-n y(x) .
$$

(iv) The Hermite polynomials $\left\{H_{n}(x)\right\}_{n=0}^{\infty}$ satisfy

$$
y^{\prime \prime}(x)-2 x y^{\prime}(x)=-2 n y(x) \text {. }
$$

In 1967, Krall and Sheffer [4] classified, as a natural generalization of classical orthogonal polynomials in one variable, all partial differential equations of the form

$$
A u_{x x}+B u_{x y}+C u_{y y}+D u_{x}+E u_{y}=\lambda u
$$

whose solutions are (weak) orthogonal polynomials in two variables, where $A, B, C$ are polynomials of degree $\leq 2, D, E$ are polynomials of degree $\leq 1$, and $\lambda$ is the eigenvalue parameter. See also [3, 6, 8] and references therein. They found ten differential equations up to a linear change of variable. Four of them have solutions, which are products of two classical orthogonal polynomials in one variable, such as Hermite product Hermite polynomials, Hermite product Laguerre polynomials, and Laguerre product Laguerre polynomials. Since Krall and Sheffer confined their study to classify the partial differential equation of the form (1.2), the products of other classical orthogonal polynomials such as Jacobi product Jacobi polynomials or Bessel product Jacobi polynomials cannot appear.

Regarding the importance of differential equation in the theory of orthogonal polynomials, we give in this paper a method to derive partial differential equations for the products of any two classical orthogonal polynomials in one variables and then find several new partial differential equations of order up to four. Lastly we explain with an example that our method can be extended to more general cases such as the product of any two sets of orthogonal functions. 


\section{Main results}

For any integer $n \geq 0$, let $\mathcal{P}_{n}$ be the space of real polynomials in two variables of degree $\leq n$. By a polynomial system, we mean a sequence of polynomials $\left\{\phi_{n-j, j}\right\}_{j=0, n=0}^{n, \infty}$ such that $\operatorname{deg}\left(\phi_{n-j, j}\right)=n, 0 \leq j \leq n$, and $\left\{\phi_{n-j, j}\right\}_{j=0}^{n}$ are linearly independent modulo $\mathcal{P}_{n-1}$ for $n \geq 0\left(\mathcal{P}_{-1}=\right.$ $\{0\})$. We let $\Phi_{n}:=\left[\phi_{n, 0}, \phi_{n-1,1}, \cdots, \phi_{0, n}\right]^{T}$ and denote the polynomial system $\left\{\phi_{n-j, j}\right\}_{j=0, n=0}^{n, \infty}$ by $\left\{\Phi_{n}\right\}_{n=0}^{\infty}$.

DeFinition 2.1. A polynomial system $\left\{\Phi_{n}\right\}_{n=0}^{\infty}$ is called an orthogonal polynomial system (OPS) if there is a Borel measure $d \mu$ such that

$$
\int \Phi_{m} \Phi_{n}^{T} d \mu=D_{n} \delta_{m n}, \quad m, n \geq 0
$$

where $D_{n}$ is a nonsingular diagonal matrix and $\delta_{m n}$ is the Kronecker delta. In this case, we say that $\left\{\Phi_{n}\right\}_{n=0}^{\infty}$ is an OPS relative to $d \mu$.

Note that if $\left\{P_{n}(x)\right\}_{n=0}^{\infty}$ and $\left\{Q_{n}(x)\right\}_{n=0}^{\infty}$ are OPS's in one variable relative to $d \mu(x)$ and $d \nu(x)$, respectively, then the product polynomial $\left\{\Phi_{n}\right\}_{n=0}^{\infty}$, where $\phi_{n-k, k}=P_{n-k}(x) Q_{k}(y)$, is an OPS relative to the product measure $d \mu(x) d \nu(y)$ in two variables. In particular, Krall and Sheffer found a second order differential equation for $\left\{H_{n-k}(x) H_{k}(y)\right\}_{k=0, n=0}^{n, \infty}$, $\left\{H_{n-k}(x) L_{k}^{(\alpha)}(y)\right\}_{k=0, n=0}^{n, \infty}$, and $\left\{L_{n-k}^{(\alpha)}(x) L_{k}^{(\beta)}(y)\right\}_{k=0, n=0}^{n, \infty}$, where $H_{n}(x)$ is the Hermite polynomial and $L_{n}^{(\alpha)}(x)$ is the Laguerre polynomial.

Here, we find differential equations for the products of any two classical OPS's. Let's first define two linear differential operators by

$$
L=a_{2}(x) \frac{\partial^{2}}{\partial x^{2}}+a_{1}(x) \frac{\partial}{\partial x}=\left(a_{22} x^{2}+a_{21} x+a_{20}\right) \frac{\partial^{2}}{\partial x^{2}}+\left(a_{11} x+a_{10}\right) \frac{\partial}{\partial x}
$$

and

$$
M=b_{2}(y) \frac{\partial^{2}}{\partial y^{2}}+b_{1}(y) \frac{\partial}{\partial y}=\left(b_{22} y^{2}+b_{21} y+b_{20}\right) \frac{\partial^{2}}{\partial y^{2}}+\left(b_{11} y+b_{10}\right) \frac{\partial}{\partial y} .
$$

Theorem 2.1. Let $\left\{P_{n}(x)\right\}_{n=0}$ and $\left\{Q_{n}(y)\right\}_{n=0}^{\infty}$ be classical OPS'S in one variable satisfying

$$
L\left[P_{n}\right]=\lambda_{n} P_{n}=n\left(a_{22}(n-1)+a_{11}\right) P_{n}
$$

and

$$
M\left[Q_{n}\right]=\mu_{n} Q_{n}=n\left(b_{22}(n-1)+b_{11}\right) Q_{n} .
$$

Then the product OPS $\left\{\Phi_{n}\right\}_{n=0}^{\infty}=\left\{P_{n-j}(x) Q_{j}(y)\right\}_{j=0, n}^{n, \infty}$ satisfies a partial differential equation: 
(a) If $a_{22}=b_{22}=0$, then

$$
b_{11} L[u]+a_{11} M[u]=a_{11} b_{11} n u .
$$

(b) If $a_{22} \neq 0$ and $b_{22}=0$, then

$$
b_{11}^{2} L[u]-a_{22} M^{2}[u]+b_{11}\left[(2 n-1) a_{22}+a_{11}\right] M[u]=b_{11}^{2} \lambda_{n} u .
$$

(c) If $a_{22}=0$ and $b_{22} \neq 0$, then

$$
a_{11}^{2} M[u]-b_{22} L^{2}[u]+a_{11}\left[(2 n-1) b_{22}+b_{11}\right] L[u]=a_{11}^{2} \mu_{n} u .
$$

(d) If $a_{22} \neq 0$ and $b_{22} \neq 0$, then

$$
\begin{aligned}
& \qquad \begin{aligned}
s_{n}^{2} M[u]= & b_{22}\left(a_{22} M-b_{22} L+b_{22} \lambda_{n}\right)^{2}[u] \\
& +\left(b_{11}-b_{22}\right) s_{n}\left(a_{22} M-b_{22} L+b_{22} \lambda_{n}\right)[u],
\end{aligned} \\
& \text { where } s_{n}=2(n-1) a_{22} b_{22}+a_{22} b_{11}+a_{11} b_{22} .
\end{aligned}
$$

Proof. We prove only (b) and (d) since the other cases can be proved by the same process. If $u(x, y):=P_{n-k}(x) Q_{k}(y)$, then $L[u]=\lambda_{n-k} u$ and $M[u]=\mu_{k} u$. If $a_{22} \neq 0$ and $b_{22}=0$, then

$$
L[u]=a_{22} k^{2} u-\left[(2 n-1) a_{22}+a_{11}\right] k u+\lambda_{n} u
$$

and

$$
M[u]=b_{11} k u .
$$

Hence, $M^{2}[u]=b_{11}^{2} k^{2} u$ and so

$$
L[u]=\frac{a_{22}}{b_{11}^{2}} M^{2}[u]-\left[(2 n-1) a_{22}+a_{11}\right] \frac{1}{b_{11}} M[u]+\lambda_{n} u,
$$

from which (b) follows. Now, assume that $a_{22} \neq 0$ and $b_{22} \neq 0$. Then we have

and

$$
L[u]=a_{22} k^{2} u-\left[(2 n-1) a_{22}+a_{11}\right] k u+\lambda_{n} u
$$

so that

$$
M[u]=b_{22} k^{2} u+\left(b_{11}-b_{22}\right) k u .
$$

where $s_{n}=2(n-1) a_{22} b_{22}+a_{22} b_{11}+a_{11} b_{22}$ and $H_{n}[u]=\left(a_{22} M-\right.$ $\left.b_{22} L+b_{22} \lambda_{n}\right)[u]$. Substituting this relation (2.5) into the equation (2.4) iteratively, we have

$$
M[u]=\frac{b_{22} H_{n}^{2}[u]}{s_{n}^{2}}+\frac{\left(b_{11}-b_{22}\right) H_{n}[u]}{s_{n}}
$$

which implies (d). 
Now, let's consider partial differential equations for each cases.

Case a. Let $a_{22}=b_{22}=0$. Then the possible $\left\{P_{n}(x)\right\}_{n=0}^{\infty}$ and $\left\{Q_{n}(y)\right\}_{n=0}^{\infty}$ are Hermite and Laguerre polynomials. Hence, there could be three cases.

Case a.1. Let $\left\{P_{n}(x)\right\}_{n=0}^{\infty}$ and $\left\{Q_{n}(y)\right\}_{n=0}^{\infty}$ be the Hermite polynomials so that $\left\{\Phi_{n}\right\}_{n=0}^{\infty}=\left\{H_{n-k}(x) H_{k}(y)\right\}_{k=0, n=0}^{n, \infty}$. Then $a_{2}(x)=1$, $a_{1}(x)=-2 x, b_{2}(y)=1$, and $b_{1}(y)=-2 y$. Hence, the differential equation (2.1) becomes

$$
u_{x x}+u_{y y}-2 x u_{x}-2 y u_{y}+2 n u=0
$$

which was found by Krall and Sheffer [4].

Case a.2. Let $\left\{P_{n}(x)\right\}_{n=0}^{\infty}$ be the Hermite polynomials and $\left\{Q_{n}(y)\right\}_{n=0}^{\infty}$ the Laguerre polynomials so that $\left\{\Phi_{n}\right\}_{n=0}^{\infty}=\left\{H_{n-k}(x) L_{k}^{(\alpha)}(y)\right\}_{k=0, n=0}^{n, \infty}$. In this case, $a_{2}(x)=1, a_{1}(x)=-2 x, b_{2}(y)=y$, and $b_{1}(y)=\alpha+1-y$. Hence, the differential equation (2.1) becomes

$$
u_{x x}+2 y u_{y y}-2 x u_{x}+2(\alpha+1-y) u_{y}+2 n u=0
$$

which was found by Krall and Sheffer [4].

Case a.3. Let $\left\{P_{n}(x)\right\}_{n=0}^{\infty}$ and $\left\{Q_{n}(y)\right\}_{n=0}^{\infty}$ be the Laguerre polynomials so that $\left\{\Phi_{n}\right\}_{n=0}^{\infty}=\left\{L_{n-k}^{(\alpha)}(x) L_{k}^{(\beta)}(y)\right\}_{k=0, n=0}^{n, \infty}$. In this case, $a_{2}(x)=x$, $a_{1}(x)=\alpha+1-x, b_{2}(y)=y$, and $b_{1}(y)=\beta+1-y$. Hence, the differential equation (2.1) becomes

$$
x u_{x x}+y u_{y y}+(\alpha+1-x) u_{x}+(\beta+1-y) u_{y}+n u=0
$$

which was found by Krall and Sheffer [4].

Case b. Let $a_{22} \neq 0$ and $b_{22}=0$. Then the possible $\left\{P_{n}(x)\right\}_{n=0}^{\infty}$ are Jacobi and Bessel polynomials, and the possible $\left\{Q_{n}(y)\right\}_{n=0}^{\infty}$ are Hermite and Laguerre polynomials. Hence, there could be four cases.

Case b.1. Let $\left\{P_{n}(x)\right\}_{n=0}^{\infty}$ be the Jacobi polynomials and $\left\{Q_{n}(y)\right\}_{n=0}^{\infty}$ the Hermite polynomials so that $\left\{\Phi_{n}\right\}_{n=0}^{\infty}=\left\{P_{n-k}^{(\alpha, \beta)}(x) H_{k}(y)\right\}_{k=0, n=0}^{n, \infty}$. In this case, $a_{2}(x)=1-x^{2}, a_{1}(x)=\beta-\alpha-(\alpha+\beta+2) x, b_{2}(y)=1$, and $b_{1}(y)=-2 y$. Hence, the differential equation (2.2) becomes

$$
4 L[u]+M^{2}[u]+2(2 n+\alpha+\beta+1) M[u]=4 \lambda_{n} u
$$

or equivalently

$$
\begin{aligned}
& u_{y y y y}-4 y u_{y y y}+2\left(2 y^{2}+2 n+\alpha+\beta-1\right) u_{y y}+4\left(1-x^{2}\right) u_{x x} \\
& -4(2 n+\alpha+\beta) y u_{y}+4[(\beta-\alpha)-(\alpha+\beta+2) x] u_{x} \\
& +4 n(n+\alpha+\beta+1) u=0 .
\end{aligned}
$$


Case b.2. Let $\left\{P_{n}(x)\right\}_{n=0}^{\infty}$ be the Jacobi polynomials and $\left\{Q_{n}(y)\right\}_{n=0}^{\infty}$ the Laguerre polynomials so that $\left\{\Phi_{n}\right\}_{n=0}^{\infty}=\left\{P_{n-k}^{(\alpha, \beta)}(x) L_{k}^{(\gamma)}(y)\right\}_{k=0, n=0}^{n, \infty}$. In this case, $a_{2}(x)=1-x^{2}, a_{1}(x)=\beta-\alpha-(\alpha+\beta+2) x, b_{2}(y)=y$, and $b_{1}(y)=\gamma+1-y$. Hence, the differential equation (2.2) becomes

$$
L[u]+M^{2}[u]+(2 n+\alpha+\beta+1) M=\lambda_{n} u
$$

or equivalently

$$
\begin{aligned}
& y^{2} u_{y y y y}+2 y(\gamma+2-y) u_{y y y}+\left(1-x^{2}\right) u_{x x} \\
& +[(\gamma+1-y)(\gamma+2-y) \\
& +(2 n+\alpha+\beta-1) y] u_{y y}+(2 n+\alpha+\beta)(\gamma+1-y) u_{y} \\
& +[(\beta-\alpha)-(\alpha+\beta+2) x] u_{x}+n(n+\alpha+\beta+1) u=0 .
\end{aligned}
$$

Case b.3. Let $\left\{P_{n}(x)\right\}_{n=0}^{\infty}$ be the Bessel polynomials and $\left\{Q_{n}(y)\right\}_{n=0}^{\infty}$ the Hermite polynomials so that $\left\{\Phi_{n}\right\}_{n=0}^{\infty}=\left\{B_{n-k}^{(d, e)}(x) H_{k}(y)\right\}_{k=0, n=0}^{n, \infty}$. In this case, $a_{2}(x)=x^{2}, a_{1}(x)=d x+e, b_{2}(y)=1$, and $b_{1}(y)=-2 y$. Hence, the differential equation (2.2) becomes

$$
4 L[u]-M^{2}[u]-2(2 n+d-1) M[u]=4 \lambda_{n} u
$$

or equivalently

$$
\begin{aligned}
& u_{y y y y}-4 y u_{y y y}+\left(4 y^{2}+4 n+2 d-6\right) u_{y y}-4 x^{2} u_{x x} \\
& -4(d x+e) u_{x}+[4 y+2(2 n+d-1)(d y+e)] u_{y}-4 n(n+d-1) u=0 .
\end{aligned}
$$

Case 6.4. Let $\left\{P_{n}(x)\right\}_{n=0}^{\infty}$ be the Bessel polynomials and $\left\{Q_{n}(y)\right\}_{n=0}^{\infty}$ the Laguerre polynomials so that $\left\{\Phi_{n}\right\}_{n=0}^{\infty}=\left\{B_{n-k}^{(d, e)}(x) L_{k}^{(\gamma)}(y)\right\}_{k=0, n=0}^{n, \infty}$. In this case, $a_{2}(x)=x^{2}, a_{1}(x)=d x+e, b_{2}(y)=y$, and $b_{1}(y)=\gamma+1-y$. Hence, the differential equation (2.2) becomes

$$
M^{2}[u]+(2 n+d-1) M[u]-L[u]+\lambda_{n} u=0
$$

or equivalently

$$
\begin{aligned}
& y^{2} u_{y y y y}+2 y(\gamma+2-y) u_{y y y}-x^{2} u_{x x} \\
& +[(\gamma+1-y)(\gamma+2-y)+(2 n+d-3) y] u_{y y} \\
& -(d x+e) u_{x}+[(2 n+d-1)(d y+e)-(\gamma+1-y)] u_{y} \\
& +n(n+d-1) u=0 .
\end{aligned}
$$

Case c. The case (c) can be obtained just by interchanging the roles of $x$ and $y$ variables in (b). 
Case d. Let $a_{22} \neq 0$ and $b_{22} \neq 0$. Then the possible $\left\{P_{n}(x)\right\}_{n=0}^{\infty}$ and $\left\{Q_{n}(y)\right\}_{n=0}^{\infty}$ are Jacobi and Bessel polynomials. Hence, there could be three cases.

Case d.1. Let $\left\{P_{n}(x)\right\}_{n=0}^{\infty}$ and $\left\{Q_{n}(y)\right\}_{n=0}^{\infty}$ be the Jacobi polynomials so that $\left\{\Phi_{n}\right\}_{n=0}^{\infty}=\left\{P_{n-k}^{(\alpha, \beta)}(x) P_{k}^{(\gamma, \delta)}(y)\right\}_{k=0, n=0}^{n, \infty}$. In this case, $a_{2}(x)=$ $1-x^{2}, a_{1}(x)=\beta-\alpha-(\alpha+\beta+2) x, b_{2}(y)=1-y^{2}$, and $b_{1}(y)=$ $\delta-\gamma-(\gamma+\delta+2) y$. Hence, the differential equation (2.3) becomes

$$
\begin{gathered}
(2 n+\alpha+\beta+\gamma+\delta+2)^{2} M[u]+\left(L-M-\lambda_{n}\right)^{2}[u] \\
+(\gamma+\delta+1)(2 n+\alpha+\beta+\gamma+\delta+2)\left(L-M-\lambda_{n}\right)[u]=0
\end{gathered}
$$

or equivalently

$$
\begin{aligned}
& \left(1-x^{2}\right)^{2} u_{x x x x}-2\left(1-x^{2}\right)\left(1-y^{2}\right) u_{x x y y}+\left(1-y^{2}\right)^{2} u_{y y y y} \\
+ & 2[\beta-\alpha-(\alpha+\beta+4) x]\left(1-x^{2}\right) u_{x x x} \\
- & 2[\beta-\alpha-(\alpha+\beta+2) x]\left(1-y^{2}\right) u_{x y y} \\
- & 2\left(1-x^{2}\right)[\delta-\gamma-(\gamma+\delta+2) y] u_{x x y} \\
+ & 2[\delta-\gamma-(\gamma+\delta+4) y]\left(1-y^{2}\right) u_{y y y} \\
+ & {\left[A_{n}\left(1-x^{2}\right)+a_{1}(x)\left(a_{1}(x)-2 x\right)\right] u_{x x} } \\
+ & {\left[B_{n}\left(1-y^{2}\right)+b_{1}(y)\left(b_{1}(y)-2 y\right)\right] u_{y y} } \\
- & 2 a_{1}(x) b_{1}(y) u_{x y}+C_{n} a_{1}(x) u_{x}+D_{n} b_{1}(y) u_{y} \\
+ & n(n+\alpha+\beta+1)(n+\gamma+\delta+1)(n+\alpha+\beta+\gamma+\delta+2) u=0
\end{aligned}
$$

where

$$
\begin{aligned}
A_{n}= & 2 n^{2}+2(\alpha+\beta+\gamma+\delta+2) n+(\gamma+\delta+1)(\alpha+\beta+\gamma+\delta+2) \\
& -2(\alpha+\beta+3) \\
B_{n}= & 2 n^{2}+2(\alpha+\beta+\gamma+\delta+2) n+(\alpha+\beta+1)(\alpha+\beta+\gamma+\delta+2) \\
& -2(\gamma+\delta+3) \\
C_{n}= & 2 n^{2}+2(\alpha+\beta+\gamma+\delta+2) n+(\gamma+\delta+1)(\alpha+\beta+\gamma+\delta+2) \\
& -(\alpha+\beta+2) \\
D_{n}= & 2 n^{2}+2(\alpha+\beta+\gamma+\delta+2) n+(\alpha+\beta+1)(\alpha+\beta+\gamma+\delta+2) \\
& -(\gamma+\delta+2) .
\end{aligned}
$$

Case d.2. Let $\left\{P_{n}(x)\right\}_{n=0}^{\infty}$ be the Jacobi polynomials and $\left\{Q_{n}(y)\right\}_{n=0}^{\infty}$ the Bessel polynomials so that $\left\{\Phi_{n}\right\}_{n=0}^{\infty}=\left\{P_{n-k}^{(\alpha, \beta)}(x) B_{k}^{(d, e)}(y)\right\}_{k=0, n=0}^{n, \infty}$. In this case, $a_{2}(x)=1-x^{2}, a_{1}(x)=\beta-\alpha-(\alpha+\beta+2) x, b_{2}(y)=y^{2}$, 
and $b_{1}(y)=d x+e$. Hence, the differential equation (2.3) becomes

$$
\left(M+L-\lambda_{n}\right)^{2}[u]+(d-1) s_{n}\left(M+L-\lambda_{n}\right)[u]-s_{n}^{2} M[u]=0
$$

or equivalently

$$
\begin{aligned}
& \left(1-x^{2}\right)^{2} u_{x x x x}+2\left(1-x^{2}\right) y^{2} u_{x x y y}+y^{4} u_{y y y y} \\
+ & 2[\beta-\alpha-(\alpha+\beta+4) x]\left(1-x^{2}\right) u_{x x x}+2\left(1-x^{2}\right)(d y+e) u_{x x y} \\
+ & \left.2[\beta-\alpha-(\alpha+\beta+2) x] y^{2} u_{x y y}+2[(d+2) y+e)\right] y^{2} u_{y y y} \\
+ & {\left[A_{n}\left(1-x^{2}\right)+a_{1}(x)\left(a_{1}(x)-2 x\right)\right] u_{x x}+\left[B_{n} y^{2}+b_{1}(y)\left(b_{1}(y)+2 y\right)\right] u_{y y} } \\
+ & 2 a_{1}(x) b_{1}(y) u_{x y}+C_{n} a_{1}(x) u_{x}+D_{n} b_{1}(y) u_{y} \\
+ & n(n+\alpha+\beta+1)(n+d-1)(n+\alpha+\beta+d) u=0,
\end{aligned}
$$

where

$$
\begin{aligned}
& A_{n}=2 n^{2}+2(\alpha+\beta+d) n+(d-3)(d+\alpha+\beta+2) \\
& B_{n}=-2 n^{2}-2(\alpha+\beta+d) n+(d+\alpha+\beta)(\alpha+\beta+1)-(d-2) \\
& C_{n}=2 n(n+\alpha+\beta+1)+(d-1)(2 n+d+\alpha+\beta+1)-\alpha-\beta-2 \\
& D_{n}=-(n+\alpha+\beta+1)((n+1) d+\alpha+\beta)-n(2 n+d+\alpha+\beta) .
\end{aligned}
$$

Case d.3. Let $\left\{P_{n}(x)\right\}_{n=0}^{\infty}$ and $\left\{Q_{n}(y)\right\}_{n=0}^{\infty}$ be the Bessel polynomials so that $\left\{\Phi_{n}\right\}_{n=0}^{\infty}=\left\{B_{n-k}^{(d, e)}(x) B_{k}^{(p, q)}(y)\right\}_{k=0, n=0}^{n, \infty}$. In this case, $a_{2}(x)=x^{2}$, $a_{1}(x)=d x+e, b_{2}(y)=y^{2}$, and $b_{1}(y)=p y+q$. Hence, the differential equation (2.3) becomes

$$
\left(M-L+\lambda_{n}\right)^{2}[u]+(p-1) s_{n}\left(M-L+\lambda_{n}\right)[u]-s_{n}^{2} M[u]=0
$$

or equivalently

$$
\begin{aligned}
& x^{4} u_{x x x x}-2 x^{2} y^{2} u_{x x y y}+y^{4} u_{y y y y}+2 x^{2}[(d+2) x+e] u_{x x x} \\
+ & 2 y^{2}[(p+2) y+q] u_{y y y}-2 x^{2}(p y+q) u_{x x y}-2(d x+e) y^{2} u_{x y y} \\
+ & {\left[A_{n} x^{2}+(d x+e)((d+2) x+e)\right] u_{x x} } \\
+ & {\left[B_{n} y^{2}+(p y+q)((p+2) y+q)\right] u_{y y}-2(d x+e)(p y+q) u_{x y} } \\
+ & C_{n}(d x+e) u_{x}+D_{n}(p y+q) u_{y} \\
+ & n(n+d-1)(n+p-1)(n+p+d-2) u=0,
\end{aligned}
$$


where

$$
\begin{aligned}
& A_{n}=-2 n^{2}-2(d+p-2) n-(p+d)(p-3), \\
& B_{n}=-2 n^{2}-2(d+p-2) n-(p+d)(d-3), \\
& C_{n}=-2 n^{2}-2(d+p-2) n-(d+p-1)(p-2), \\
& D_{n}=-2 n^{2}-2(d+p-2) n-(d+p-1)(d-2) .
\end{aligned}
$$

Lastly we give one more example which implies that our method can be extended to a more general case such as the product of two sets of orthogonal functions. Consider a sequence of orthogonal functions $\left\{f_{n}(x)\right\}_{n=0}^{\infty}$, where $f_{n}(x)=\cos n x$. Then $f_{n}(x)$ is an eigenfunction of the Sturm-Liouville differential equation

$$
f_{n}^{\prime \prime}(x)+n^{2} f_{n}(x)=0
$$

Now, let $\phi_{n-k, k}=f_{n-k}(x) H_{k}(y), 0 \leq k \leq n$. Then it can be easily shown that $\left\{\Phi_{n}\right\}_{n=0}^{\infty}$ is a sequence of orthogonal functions in two variables relative to $H\left(\pi^{2}-x^{2}\right) e^{-y^{2}} d x d y$, where $H(x)$ is the Heaviside step function. More precisely,

$$
\int_{-\infty}^{\infty} \int_{-\pi}^{\pi} \Phi_{n} \Phi_{m}^{T} e^{-y^{2}} d x d y=\operatorname{diag}\left[d_{0}, d_{1}, \cdots, d_{n}\right] \delta_{m n}, n, m=0,1,2, \cdots
$$

where

$$
d_{k}=\int_{-\pi}^{\pi} f_{n-k}^{2}(x) d x \int_{-\infty}^{\infty} H_{k}^{2}(y) e^{-y^{2}} d y, \quad k=0,1, \cdots, n .
$$

Now, let $u(x, y)=f_{n-k}(x) H_{k}(y)$. Then from the equations (1.1) and (2.6), we can see that

$$
u_{y y}-2 y u_{y}+2 k u=0
$$

and

$$
u_{x x}+(n-k)^{2} u=0 .
$$

Hence, the sequence of orthogonal functions $\left\{\Phi_{n}\right\}_{n=0}^{\infty}$ satisfies

$$
u_{y y y y}-4 y u_{y y y}+4 u_{x x}+4\left(y^{2}+n-1\right) u_{y y}-4 y(2 n-1) u_{y}+4 n^{2} u=0 .
$$

We emphasize with this example that our method can be extended a more general case. 


\section{References}

[1] S. Bochner, Über Sturm-Liouvillesche Polynomsysteme, Math. Z. 29 (1929), 730736.

[2] T. S. Chihara, An Introduction to Orthogonal Polynomials, Gordon and Breach, New York, 1977.

[3] T. H. Koornwinder, Two-variable analogues of the classical orthogonal polynomials, Theory and Applications of Special Functions, R. Askey Ed., Academic Press, 1975, 435-495.

[4] H. L. Krall and I. M. Sheffer, Orthogonal polynomials in two variables, Ann. Mat. Pura Appl. 76 (1967), 325-376.

[5] K. H. Kwon, D. W. Lee, and L. L. Littlejohn, Differential equations having orthogonal polynomial solutions, J. Comput. Appl. Math. 80 (1997), 1-16.

[6] __ Orthogonal polynomial eigenfunctions of second-order partial differential equations, Trans. Amer. Math. Soc. 353 (2001), 3629-3647.

[7] K. H. Kwon and L. L. Littlejohn, Classification of classical orthogonal polynomials, J. Korean Math. Soc. 34 (1997), 973-1008.

[8] L. L. Littlejohn, Orthogonal polynomial solutions to ordinary and partial differential equations, in Proc. 2nd International. Symp. Orthogonal Polynomials and their Applications, M. Alfaro et al. I(Eds), Lecture Notes Math. 1329, Springer, 1988, 98-124.

[9] G. Szegö, Orthogonal Polynomial, Amer. Math. Soc. Colloq. Publ. 23 (1975)

Department of Mathematics, Teachers College, Kyungpook National UniVERSITY, TAEGU 702-701, KOREA

E-mail: dongwon@mail.knu.ac.kr 